\title{
Research on Online Learner Modeling and Course Recommendation Based on Emotional Factors
}

\author{
Yamin Wang $\mathbb{D}^{1,2}$ \\ ${ }^{1}$ Changsha Normal University, Changsha 410100, Hunan, China \\ ${ }^{2}$ Hunan Provincial Research Institute of Education, Changsha 410105, Hunan, China \\ Correspondence should be addressed to Yamin Wang; wangyamin@csnu.edu.cn
}

Received 23 November 2021; Revised 29 December 2021; Accepted 3 January 2022; Published 4 February 2022

Academic Editor: Sikandar Ali

Copyright (c) 2022 Yamin Wang. This is an open access article distributed under the Creative Commons Attribution License, which permits unrestricted use, distribution, and reproduction in any medium, provided the original work is properly cited.

With the popularization of online education and the idea of learning anytime and anywhere, more and more learners search and learn courses of various disciplines through online learning platforms to meet their personal knowledge needs. With the increase of the number of courses, it is difficult for learners to find the courses they want quickly and accurately; that is, they encounter the problems of information overload and cognitive maze. Therefore, how to recommend personalized courses for learners according to their preferences has become one of the important problems that need to be solved urgently to improve the service quality of courses in online learning platforms. Therefore, in order to improve the accuracy of course recommendation, it is necessary to build an accurate and complete learner model. In order to improve the application effect of recommendation, this paper focuses on the recommendation method of emotional factors to improve the recommendation efficiency of learning resources. The traditional recommendation model is a method based on the user's purchase behavior and historical information. However, in the emotional factors, the effect of traditional recommendation is limited. This paper proposes a recommendation method based on emotional factors, which may consider the emotional and psychological factors of scholars according to the learning content of learners. The experimental results show that the learner model incorporating learners' affection can reflect learners' preferences more accurately, and the use of deep neural factorization machine for curriculum recommendation can effectively improve the accuracy of curriculum recommendation.

\section{Introduction}

Thanks to the rapid development of information technology such as the Internet of Things [1-5] and the spread of educational ideas that emphasize the dominant position of learners, a large number of online courses have emerged since 2008. With the freedom of curriculum selection, flexible learning time arrangement, diversified learning methods, and abundant curriculum resources, they are favored by more and more learners. Nowadays, there are countless and abundant online courses, but it is very difficult for learners to find the courses they are interested in in a large number of online course resources. Information overload, cognitive maze, and other problems are very serious, which bring a lot of inconvenience to learners' learning. Recommendation system is one of the methods to solve information overload. Benefiting from the large amount of educational data and information generated by learners on the online learning platform, the enhancement of computer ability, and the continuous growth of largescale online courses, personalized course recommendation came into being, which also attracted more and more people's attention and helped learners to provide convenience for personalized learning. Therefore, the key point is to improve the accuracy of course recommendation.

With the development of deep learning, its function is stronger than the traditional model based on Markov chain and factorization, which is the key factor affecting the performance of deep learning model [6]. The hybrid model integrates RNN and LSTM [7] and provides better results than existing models in terms of accuracy. Literature [8] uses the performance of big data artificial intelligence platform 
for qualitative purposes and shows the practical literature as the future algorithm experiment and system joint design benchmark. Literature [9] aims at the traditional collaborative filtering technology, such as matrix decomposition. It uses linear combination to analyze potential learners and projects according to learners' preferences. Deep learning is one of the effective methods to construct a recommendation system [10]. Literature [11] introduces the causal relationship between learner characteristics, learning experience, and test scores. The results of modeling technology show that the key factors and their contribution to test scores can be measured. Literature [12] aims at the shortage of lecturers caused by large-scale online courses and the difficulty of learners' choice, and it is difficult to find courses suitable for individuals, so learners' learning progress is slow. The e-learning recommendation framework based on deep learning [13] has the function of learning in large-scale data. The evaluation and prediction method of deep learning is easy to implement and will not disclose any personal information. Learners prefer to be able to use similarity relations for analysis [14] so as to improve the recommendation accuracy. With the continuous growth of the amount, complexity, and dynamics of online information [15], the recommendation system provides personalized recommendation by retrieving the most relevant information and services from a large amount of data. It is an effective key solution to solve the increasing problem of information overload. Thanks to the innovative progress of deep learning, people apply it to recommendation system [16]. Different from the traditional recommendation model, deep learning can collect nonlinear and important user project relationships and can use higher-level data to digitize complex abstract code. In the era of big data, evaluating the characteristics of a large amount of information to make choices is a tedious and time-consuming task [17]. One solution to alleviate this overload problem is to build a recommendation system that can process a large amount of data and support users' decision-making ability. Personalized recommendation system [18] is a way for current learning systems to realize learners' learning ideas. Based on the combination of MF and MLP [19], the prediction effect is optimized, and the inherent product limitation of model expression ability is eliminated. Compared with the existing benchmark algorithms, the model improves the effectiveness of recommendation, and the deep neural system [20] effectively solves the complex problems of artificial intelligence. In the related research work, the deep learning model is used to recommend related resources, and other related similar users are recommended. The recommendation of such features is a method implemented based on user purchase behavior and history-related information. In the emotional factors, the effect of this recommendation is limited. This paper proposes a recommendation method based on emotional factors, which may consider the emotional and psychological factors of scholars according to the learning content of learners. In the second part, the emotional characteristics of learners are studied in depth; in the third part, the theoretical knowledge and recommendation technology of deep learning are explained; in the fourth part, an experimental comparison is made between the deep learning model with emotion fusion and other methods.

\section{Theory}

2.1. Learner Characteristics. Learners are the object and subject of all educational activities. In a narrow sense, learners refer to those who receive knowledge in school, that is, students. With the development of science and technology, nonschool students use online courses to increase their learning knowledge and improve their self-skills. The concept of learners has also become more extensive. Educated people are collectively called learners. With the expansion of learners' conceptual scope, learners' own characteristics, namely, consciousness, autonomy, and creativity, are also more remarkable. And under the influence of the concept of lifelong learning, learners have great development possibility and plasticity, there is a need for continuous development, and all these have caused great changes in learners' learning motivation, emotion, and needs. Compared with traditional learners, learners' learning motivation is more clear and strong, so their learning attitude begins to change from passive learning emotional attitude to positive learning emotional attitude. At the same time, learners' needs for their own development are more urgent, and the connotation of learning needs is richer. These changes of learners also cause a series of changes in learner characteristics. The characteristics of learners in traditional education often only include the demographic characteristics of learners, It is relatively simple, but the current learners have rich characteristics, including learners' psychological, physiological, and external environment factors in the learning process, including learners' age, gender, major, education level, learning attitude, learning motivation, learning expectation, learning emotion, cognitive level, learning style, economic and social background, and other factors.

Recommendation based on the learner model is an effective way to improve the accuracy of recommendation. Current learner models mainly include the following categories: (1) coverage model: this model only describes students' cognitive state but lacks the description of learners' learning behavior and learning emotion; (2) bias model: this model builds a database of learners' knowledge processing errors to improve learners' performance in the test; (3) cognitive model: this model makes up for the deficiency of the first two models. It focuses not only on the cognitive state of learners but also on the study of learners' emotions, attitudes, and learning styles. It has been widely used in the field of personalized learning, but the lack of learners' emotion has always been an urgent problem to be solved in the cognitive model.

Learner emotion is one of the important characteristics of learners, which has a decisive influence on the accuracy of the learner model. However, the existing emotion analysis of learners' comments regards the whole emotion of comments as an emotional polarity but turns a blind eye to the various emotions of learners in comments. Therefore, the excavated emotion of learners cannot accurately depict learners' 
preferences, and the learner model constructed by this method cannot play a positive role in curriculum recommendation. In order to improve the accuracy of course recommendation, it is necessary to extract the affective information of learners' course reviews in order to build an accurate and perfect learner model. The long-term and short-term memory network based on attention mechanism (AT-LSTM) [21] can effectively capture learners' emotional attitudes towards specific aspects of the curriculum reviewed in the article, and the Deep Neural Decomposition Machine (DeepFM) can simulate the interaction between features, which is better for processing high-dimensional sparse data. Therefore, this paper uses AT-LSTM to mine learners' emotions, constructs a learner model integrating learners' emotions, and then uses DeepFM to recommend courses.

2.2. Learner Emotion. Learner's emotion refers to all kinds of emotional expressions produced by learners in their learning process, so it can accurately show learners' learning preferences and is one of the important characteristics of learners. If the learner's emotion on the course is positive, it shows that the learner prefers this kind of course. On the contrary, if it is negative, it shows that the learner does not like this kind of course. Therefore, if we can tap learners' emotions, we can describe learners' preferences in more detail and provide them with personalized learning support services so as to accurately improve learners' learning efficiency. Generally, online learners' emotions are hidden in learners' text comments, messages, questions, forum interactions, barrage, and so on, and learners' preference information can be obtained by emotional mining of this information. The emotional information can not only be used in the field of personalized learning service but also adjust teaching and make a benign intervention on learning according to learners' emotional state.

\subsection{Initializing Online Learner Model with Emotional Factors.} The initialization of the learner model is mainly to formalize the static information in learner information, which is usually the basic personal information filled in by learners when registering, the scale for investigating learning style, and the test questions related to learning content. Through initialization, the learner model is designed by using the characteristics of learners, such as basic information and learning preferences. Other pieces of related information, such as learners' emotions and learning behaviors, need to be processed more intensively before building learner models. The learner model is the abstract embodiment of learner characteristic information. Therefore, it is necessary to use learner characteristics for formal definition. The following are the formal definitions of learner characteristics in the five dimensions involved in this paper.

2.3.1. Basic Personal Information. The personal basic information in this paper includes basic attribute information and static attribute information related to learning, which can be obtained when learners register and will not change with the learning process. The formal definition of personal basic information is

Personal - Information

$=\{I D$, Age, Sex, Profession, Education, Research - Direction $\}$,

where ID, Age, Sex, Profession, Education, and ResearchDirection, respectively, indicate the identity number, age, gender, major, educational background, and research direction of learners.

2.3.2. Emotional Attitude. This paper mainly focuses on learners' emotion towards curriculum, so we define learners' emotion setting towards curriculum as a multidimensional vector, which is formalized as

$$
\begin{aligned}
\text { Aspect }- \text { Emotion }= & \left\{\left(A_{1}, E_{1}, \alpha_{1}\right),\left(A_{2}, E_{2}, \alpha_{2}\right),\right. \\
& \ldots \ldots\left(A_{i}, E_{I}, \alpha_{i}\right) \mid i \in[1,2, \ldots \ldots N],
\end{aligned}
$$

where $A_{n}$ denotes the learners' comments on the nth aspect of the course, $E_{n}$ denotes the learners' category emotion on the nth aspect of the course, and $\alpha_{n}$ is the weight vector calculated by $E_{n}$ in AT-LSTM, which is used to express the intensity of aspect emotion.

Cognitive level this paper will judge learners' recognition by the scores of chapter tests, overall course tests, and whether they have obtained certificates of completion.

As for the index of knowledgeability, this paper does not consider the factors of curriculum types for the time being.

$$
\begin{aligned}
& \text { Cognitive-Level } \\
& \quad=\left\{I D, G_{i} \text {, Grade, Certificate } i \in[1,2, \ldots \ldots N]\right\},
\end{aligned}
$$

where $G_{i}$ represents the test scores of Chapter $i$, Grade represents the test scores of the whole course, Certificate indicates whether the learner has obtained the certificate of completion; when its value is 1 , it means that the learner has obtained the certificate of completion, and when it is 0 , it means that the learner has not obtained the certificate of completion.

Learning behavior can show learners' learning preference to a certain extent, which is the information left by learners on the online learning platform. In this paper, the learning behavior in learners' characteristics is formally defined as

$$
\begin{aligned}
& \text { Learning - Behavior } \\
& \quad=\{I D, C R T, L L T, N O C L C, L O V, N C C, N P D\},
\end{aligned}
$$

where ID learner's identification number, CRT, LLT, NOCLC, LOV, NCC, and NPD, respectively, indicate learner's course registration time, last landing time, number of course study chapters, visit time, number of course study chapters, and number of participation in discussion. Through the statistics and analysis of this information, it can reflect the degree of learners' love for the course to a certain extent. 
The learner's style shows the learner's preferred learning style, and its acquisition method is generally collected through the scale when the learner registers in the system. In this paper, learning methods are divided into initiative, reflection, intuition, sensory, sequence, and synthesis. Active learners like collaborative learning. Reflective learners, on the contrary, like to think alone. Intuitive learners like innovation and hate repetition. Sensory learners like learning resources such as video and audio. Sequential learners tend to follow the logic to learn. Comprehensive learners like to learn by jumping and then digest it slowly. Its formal definition is

$$
\begin{aligned}
& \text { Learning - Styl } \\
& \left.\left.\left.\left.\left.\qquad=\left\{D_{1}, S_{1}, D_{2}, S_{2}\right),\left(D_{3}, S_{3}\right)\right),\left(D_{4}, S_{4}\right)\right),\left(D_{5}, S_{5}\right)\right),\left(D_{6}, S_{6}\right)\right)\right\} .
\end{aligned}
$$

where $D_{1}, D_{2}, D_{3}, D_{4}, D_{5}, D_{6}$, respectively, represent six learning styles and $S_{1}, S_{2}, S_{3}, S_{4}, S_{5}, S_{6}$, respectively, represent the values of learners in the six types. The maximum value is selected as the corresponding learning style of learners and will not be changed after that.

\section{Technology}

3.1. Factorizer. Factorization machine [22] is a general factor model based on matrix factorization proposed by Steffen Rendle. It belongs to the hidden factor model [23], which uses the interaction between different feature vectors to model, predicts the user's score on items, and then recommends them. Factorization machine is a collaborative filtering recommendation technology based on model. Different from traditional, it has strong scalability and flexible feature transformation. Not only can it deal with complex data simply and accurately, but also its computational complexity will not increase with the increase of data volume. It is very effective in dealing with the sparse problem of high-dimensional data.

For an $n$-dimensional eigenvector $x, \quad x=\left(x_{1}\right.$, $\left.x_{2}, \ldots, x_{n}\right) \in R^{n}$, where $x=\left(x_{1}, x_{2}, \ldots, x_{n}\right) \in R^{n}$ are not independent of each other, $y_{i}$ is the predicted value of the corresponding target, and when $n=2$, the factorization machine model expression is as follows:

$$
\widehat{y}(x)=w_{0}+\sum_{i=1}^{n} w_{i} x_{i}+\sum_{i=1}^{n} \sum_{j=i+1}^{n}\left\langle v_{i}, v_{j}\right\rangle x_{i} x_{j},
$$

where $w_{0} \in R$ and $w \in R^{n}$ denote the interaction of eigenvectors $x_{i}$ and $x_{j}$, coefficient matrix $V \in R^{n \times k},\left\langle v_{i}, v_{j}\right\rangle$ is the dot product of the vectors $v_{i}$ and $v_{j}$ of size $k$, and the expression is

$$
\left\langle v_{i}, v_{j}\right\rangle=\sum_{f=1}^{k} v_{i, f} \cdot v_{j, f} .
$$

Here, $k \in N^{+}$is the hyperparameter that defines the decomposition dimension, and $v_{i, f}, v_{j, f}$ are the hidden vectors corresponding to the eigenvectors $x_{i}$ and $x_{j}$, respectively.
The methods to optimize the model parameters are random gradient descent method, least square method, and Markov chain Monte Carlo method.

Factorization machine can use matrix to decompose the input high-dimensional data into low-order matrix and use the product of sum to predict and model. It can also improve the accuracy and efficiency of recommendation.

3.2. Deep Learning. Neural networks [24] are an abstract model that imitates the performance of biological neural networks to the outside world. The main function is the mathematical model of distributed parallel processing algorithm for information. Which is formed by connecting a large number of different neurons with each other. The connection between two neurons represents the weight. There are several basic parts: input layer, hidden layer, and output layer. Its basic principle is that neurons receive information through the outside world and transmit information by weighted connection. The input value is compared with the threshold value of neuron, the message is processed, and the output is generated. Neural network has strong learning ability, representation ability, and the ability to choose the optimal solution like human beings when encountering problems and can effectively deal with nonlinear data. The algorithm has strong adaptability and high efficiency. BP neural network [25] is a typical feedforward neural network. Figure 1 is a classical BP neural network structure diagram. The figure shows the input layer, output layer, and hidden layer, and the link weight between each neuron, where $x_{d}$ represents the input neuron, $b_{q}$ represents the hidden layer neuron, $y_{i}$ represents the output neuron, $w_{q j}$ represents the connection weight between the output layer neuron and the hidden layer, and $v_{d h}$ represents the connection weight between the input layer neuron and the hidden layer. The gradient descent method (GD) is used to solve the optimization of model parameters by the BP neural network.

The complexity of the model is increased by increasing the number of hidden layers, and the data will be processed layer by layer. Therefore, it can improve the learning ability of the model and reduce the risk of overfitting. The common training method of deep learning is to use unsupervised layer-by-layer training. Its basic principle is to train one hidden layer at a time and then assign the output result of this layer to the input of the next hidden layer. After training, adjust the whole neural network to achieve the optimal model state; another effective training method is weight sharing; that is, a group of neurons share a connection weight. Compared with Shenwang, the feature representation ability of deep learning is stronger, more efficient, more accurate, and more adaptable. Common deep learning models include recurrent neural network (RNN), long-term and short-term memory neural network (LSTM) [26, 27], and deep belief network (DBN) [28].

Recurrent neural network (RNN) [29] is an extension of traditional feedforward neural network. It has the memory function of inputting information. With the increase of neural network layers, the gradient of standard RNN will 


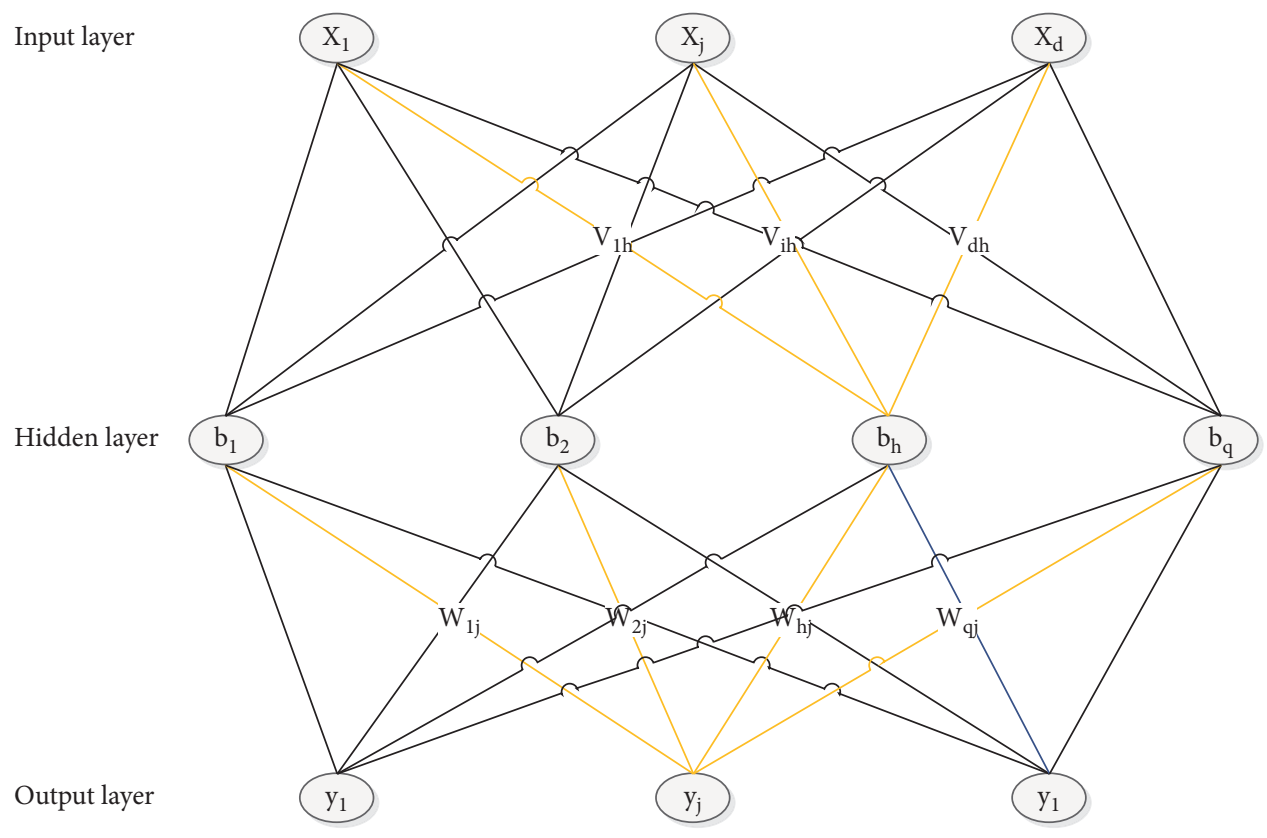

Figure 1: Structure diagram of BP neural network.

disappear or explode. To overcome these problems, the Long-term Short-term Memory Network (LSTM) is proposed. LSTM is a special kind of RNN, and its structure is shown in Figure 2. It represents word vectors and hidden vectors in sentences. In an LSTM architecture, there are three gates: a forget gate, an input gate, an output gate, and a cell memory state. The red circle represents the bitwise operation of elements, the yellow matrix is the learned neural network layer, and the top horizontal arrow represents the cell state, on which the information is not easy to change. The first forgetting gate is responsible for deleting some useless information, and the next step is to determine what new information can be stored in the cell state. This is divided into two parts. First, the input gate layer determines what value will be updated. Then, the tanh layer creates a new candidate value vector and adds it to the state. The next step is to update the cell state. Finally, the output gate establishes the output information based on the cell state.

$$
f_{i}=\sigma\left(w_{f}\left[x_{i}, h_{i-1}\right]+b_{f}\right) .
$$

Formula (8) is the mathematical expression of the forget gate, which reads $x_{i}$ and $h_{i-1}$ and outputs a value between 0 and 1 to each number in the cell state $c_{i-1} .1$ means "completely retained," and 0 means "completely discarded."

$$
\begin{aligned}
I_{i} & =\sigma\left(w_{I}\left[x_{i}, h_{i-1}\right]+b_{I}\right), \\
\widetilde{C} & =\tan h\left(W_{c}\left[x_{i}, h_{i-1}\right]+b c .\right.
\end{aligned}
$$

Formulas (9) and (10) represent $\widetilde{C}$ new candidate value vector, and $I_{i}$ determines what value has been updated.

$$
C_{i}=f_{i} * C_{i-1}+I_{i} * \widetilde{C}_{i} .
$$

Formula (11) indicates that the cell state is updated from $f_{i}$ to $C_{i}$, and $f_{i} * C_{i-1}$ indicates that the information determined to be discarded in the previous step, which is a new candidate value vector, is discarded.

$$
\begin{aligned}
& o_{i}=\sigma\left(W_{0}\left[x_{i}, h_{i-1}\right]+b_{0},\right. \\
& h_{i}=o_{i} * \tan h\left(C_{i}\right) .
\end{aligned}
$$

Formula (12) represents the state of the unit to be output, which is then processed by tanh in formula (13) to obtain a value between -1 and 1 , which is finally multiplied by the active gate layer to determine the part to be output in Figure 2.

In recent years, due to the powerful function of deep learning, it has played an important role in the field of personalized learning. It is very accurate to paint learners through deep learning technology, so the application of deep learning in learner modeling can make learner models more perfect, while the application in personalized resource recommendation can effectively improve the accuracy of recommendation.

\subsection{Online Course Recommendation Method Combined with} Learners' Emotional Factors. With more and more learners joining online courses, it is inevitable to face more and more difficulties to provide better personalized services for learners. Constructing a perfect learner model can not only improve the accuracy of curriculum recommendation and the utilization rate of curriculum resources but also provide a basis for teachers' teaching decision-making, teaching tool optimization, and self-assessment, help learners learn better, and promote the personalized development of online education. Based on this, a learner model combining emotional factors is built, and then a deep neural factorization machine is used to recommend courses. The course recommendation framework is shown in Figure 3. 


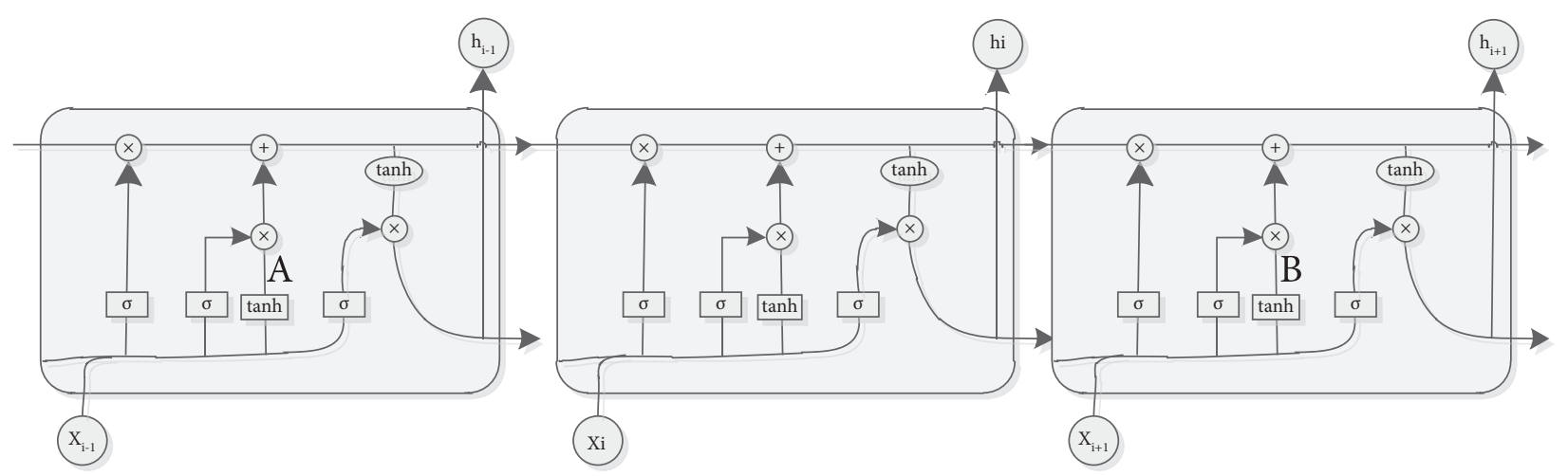

Figure 2: Structure diagram of standard LSTM.

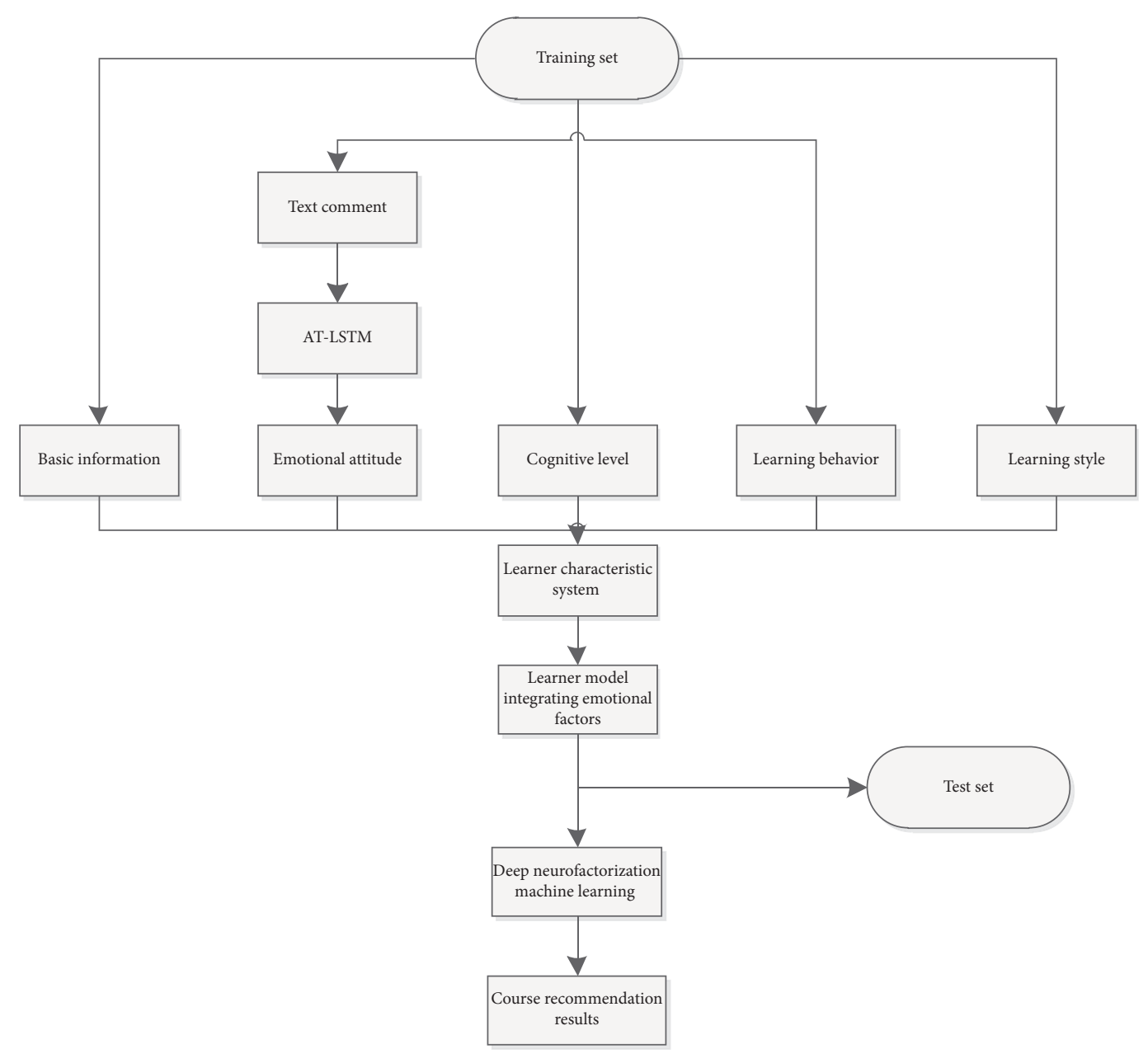

Figure 3: Curriculum recommendation framework.

3.4. Deep Factorization Machines (DeepFM). Deep Factorization Machines (DeepFM) was proposed by Guo et al. DeepFM is a combination of FM and DNN (deep neural network). It can not only simulate low-order feature interactions such as FM but also simulate high-order feature interactions such as DNN. DeepFM can conduct end-to-end training without any functional engineering. Figure 4 is the architecture diagram of DeepFM. As you can see from Figure 4, the FM component and the Deep component share the same input. Its training model is

$$
\widehat{y}=\operatorname{sigmoidy}_{\mathrm{FM}}+y_{\mathrm{DNN}} \text {. }
$$

where $y_{\mathrm{FM}}$ is the output result of the FM component, $y_{\mathrm{DNN}}$ is the output result of the Deep component, and $\hat{y} \epsilon(0,1)$ is the 


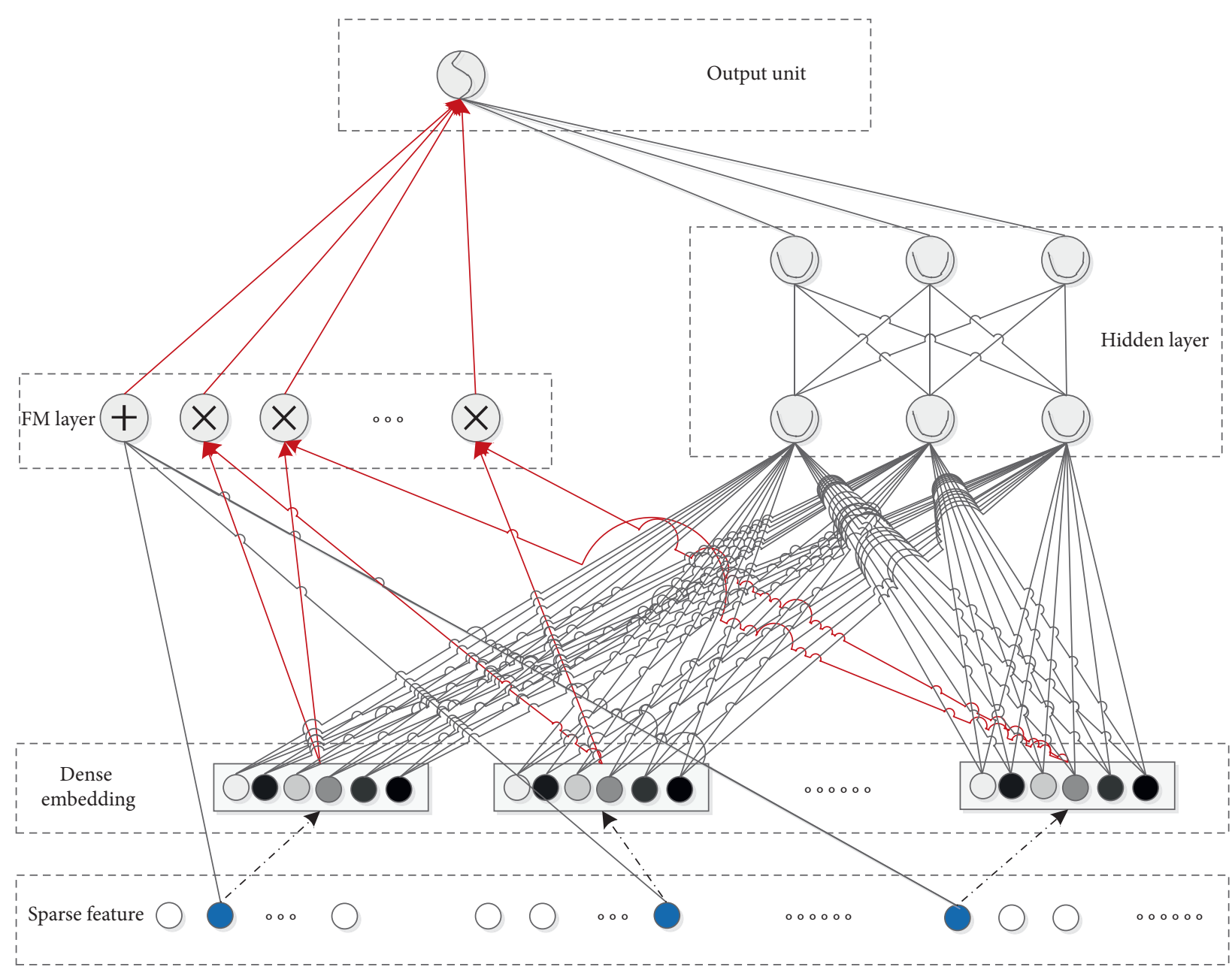

Characteristic i

Feature J

000000

Characteristic $m$
(十) Addition
X) Inner product
(S) Sigmodl function
Activation function

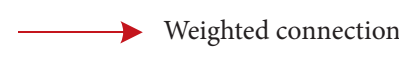

Ordinary connection

Figure 4: DeepFM architecture diagram.

prediction result of DeepFM. The expression for $y_{\mathrm{FM}}$ is as follows:

$$
y_{\mathrm{FM}}=w, x+\sum_{i=1}^{d} \sum_{j=i+1}^{d} V_{i}, V_{j} x_{i} \cdot x_{j},
$$

where $w, x$ are the first-order features and the inner product is the second-order cross feature. The expression for $y_{\mathrm{DNN}}$ is as follows:

$$
\begin{aligned}
& a^{(l+1)}=\sigma\left(w^{(l)} a^{(l)}+b^{(l)},\right. \\
& y_{\mathrm{DNN}}=W^{|H|+1} \cdot a^{|H|}+b^{|H|+1},
\end{aligned}
$$

where $l$ represents the number of layers of DNN, $\sigma$ is the activation function, $w^{(l)}$ represents the weight of DeepFM, $a^{(l)}$ represents the output of layer $l, b^{(l)}$ is the offset term, and $|H|$ represents the hidden layer. After the training of formula (15) is completed, a low-dimensional dense vector is generated, and finally, the predicted value is generated via a sigmoid activation function.

The parameters of the deep neural factorizer are transmitted from the input layer to the hidden layer. If there is an error, it will adjust in the opposite direction until the error is within the allowable range, and then it will be transmitted through the hidden layer output layer. After adjusting the existing errors as before, the output results are 
output, and the weights between different grades are constantly adjusted in multiple training. The parameters of the model are also trained in this way, thus completing the data learning of the model. The average loss function can be used to optimize the model parameters:

$$
L_{\text {reg }}=\sum_{X \in D}\left(\widehat{r}_{u, i}(x)-r_{u, i}(x)\right)^{2} \text {, }
$$

where $D$ represents the training set and $r_{u, i}(x)$ represents the user's overall score for item $i$.

\subsection{Deep Neural Factorization Machine Input Vector} Representation. Feature selection has a great influence on the accuracy of recommendation. Based on the constructed learner model, this paper recommends learners' age, gender, major, educational background, research direction, learning behavior, learning style, cognitive level, curriculum popularity, curriculum keywords, and scores. The popularity of courses is defined by curriculum scores, the number of participants in courses, and the number of participants in scores. The formula is as follows:

$$
\text { Popularity }(i)=\mathrm{AS}_{i} * \frac{\mathrm{NP}_{i}}{\mathrm{CP}_{i}} .
$$

Popularity $(i)$ denotes the popularity of course $i, \mathrm{AS}_{i}$ denotes the average score of course $i, \mathrm{NP}_{i}$ denotes the number of people participating in scoring, and $\mathrm{CP}_{i}$ denotes the number of people signing up for the course.

Curriculum keywords reflect learners' preference for curriculum resources. Course names are often concise and generalized, which cannot accurately reflect learners' learning preferences. For example, learners are interested in data structure courses, but there are $C$ language versions and $\mathrm{C}++$ versions of data structure courses. Course names often cannot reflect learners' preferences in detail, and course profiles can reflect learners' preferences in detail. In this paper, TF * IDFalgorithm is used to extract course keywords from course introduction. This algorithm is mainly used to distinguish the importance of words to documents. If the frequency of occurrence in other documents is low, the word is determined to have better category discrimination and assigned a higher weight. It is expressed as a vector: $W_{i, n}=\left\{w_{i 1}, w_{i 2}, w_{i 3} \ldots w_{i, n}\right\}$.

$W_{i, n}$ represents the weight of the $n$th keyword in the introduction of course $i$. The objective function can be expressed as

$$
\text { 㐱: } \begin{aligned}
U \times C \times B_{1} \times \cdots \times B_{5} \times C_{1} \times \cdots \times C_{6} \\
\quad \times S \times T_{1} \times \cdots \times T_{n} \times w_{t 1} \times \cdots \times w_{t n} \times S E_{1} \\
\quad \times \cdots S E_{i} \times C l_{i} \times \cdots \times C l_{3} \times P \longrightarrow R,
\end{aligned}
$$

where $U$ stands for learner space, $C$ represents the curriculum resource space, $B_{1} \sim B_{5}$ denote age, sex, major, research direction, and educational background, $C_{1} \sim C_{6}$ denote the course registration time, the last landing time, the number of course study chapters, the visiting time, the number of course study chapters, and the number of participating discussions, $\mathrm{S}$ denotes learning style, $T_{1} \sim T_{n}$ denote the text space of course introduction, $w_{t 1} \sim w_{t n}$ are the weight space of $t_{1} \sim t_{n}, \mathrm{SE}_{1} \sim \mathrm{SE}_{i}$ denote learners' feelings about course difficulty, course content, teacher's explanation ability, and course expression form, and $\mathrm{Cl}_{1}, \mathrm{Cl}_{2}$, and $\mathrm{Cl}_{3}$ indicate the chapter test score, course test score, and whether the certificate is obtained. $P$ denotes the popularity of the course, and $R x(u, i)=$ denotes the scoring space of the user. Accordingly, the input eigenvector of DeepFM is constructed:

$$
\begin{aligned}
x(u, i)= & \left\{(u, 1),(c, 1),\left(b_{j}, 1\right),\left(c_{j}, 1\right),(S, 1),\left(t_{m}, 1\right)\right. \\
& \left.\left(w_{m}, r\right),\left(S E_{k}, 1\right),\left(c l_{q}, 1\right),(p, 1)\right\},
\end{aligned}
$$

where $\quad i=1 \ldots 5, j=1 \ldots 6, m=1 \ldots n, k=1 \ldots n$, $q=1 \ldots 3, r \in[0,1]$.

\section{Experiment and Analysis}

The learner model considers five dimensions of features that affect learners' curriculum recommendation and thinks that it is not a single feature that affects learners' curriculum selection, so deep neural factorization machine is used for curriculum recommendation. In order to verify the effectiveness of the method proposed in this paper, $80 \%$ of the data set is selected as the training set and $20 \%$ as the test set, the model is learned through the training set, and finally, the test set is used for verification.

\subsection{Selection of Curriculum Recommendation Evaluation} Index. The widely used evaluation index in the recommended field is prediction accuracy. The accuracy indicators used in this paper are root mean square error (RMSE) and mean absolute error (MAE). The RMSE representation is the sum of squares of the deviation between the predicted value and the true value and the square root of the ratio of the sample set $m$, defined as

$$
\operatorname{RMSE}(x, h)=\sqrt{\frac{1}{m} \sum_{i=1}^{m}}\left(h\left(x^{(i)}\right)-y^{(i)}\right)^{2} .
$$

MAE represents the ratio of the absolute value of deviation from the arithmetic mean of all individual predictions to the predicted number $m$, which is defined as

$$
\operatorname{MAE}(x, h)=\frac{1}{m} \sum_{i=1}^{m}\left|h\left(x^{(i)}\right)-y^{(i)}\right|,
$$

where $h\left(x^{(i)}\right)$ is the actual score value and $y^{(i)}$ is the predicted score value. The smaller the values of RMSE and MAE, the higher the accuracy of model recommendation.

\subsection{Deep Collaborative Filtering Recommendation Model} Algorithm. A variety of deep collaborative filtering recommendation model algorithms, such as GMF, EGMF, MLP, NeuMF, and IUNCF, are tested on data sets. The model prediction factor in each algorithm is uniformly set 

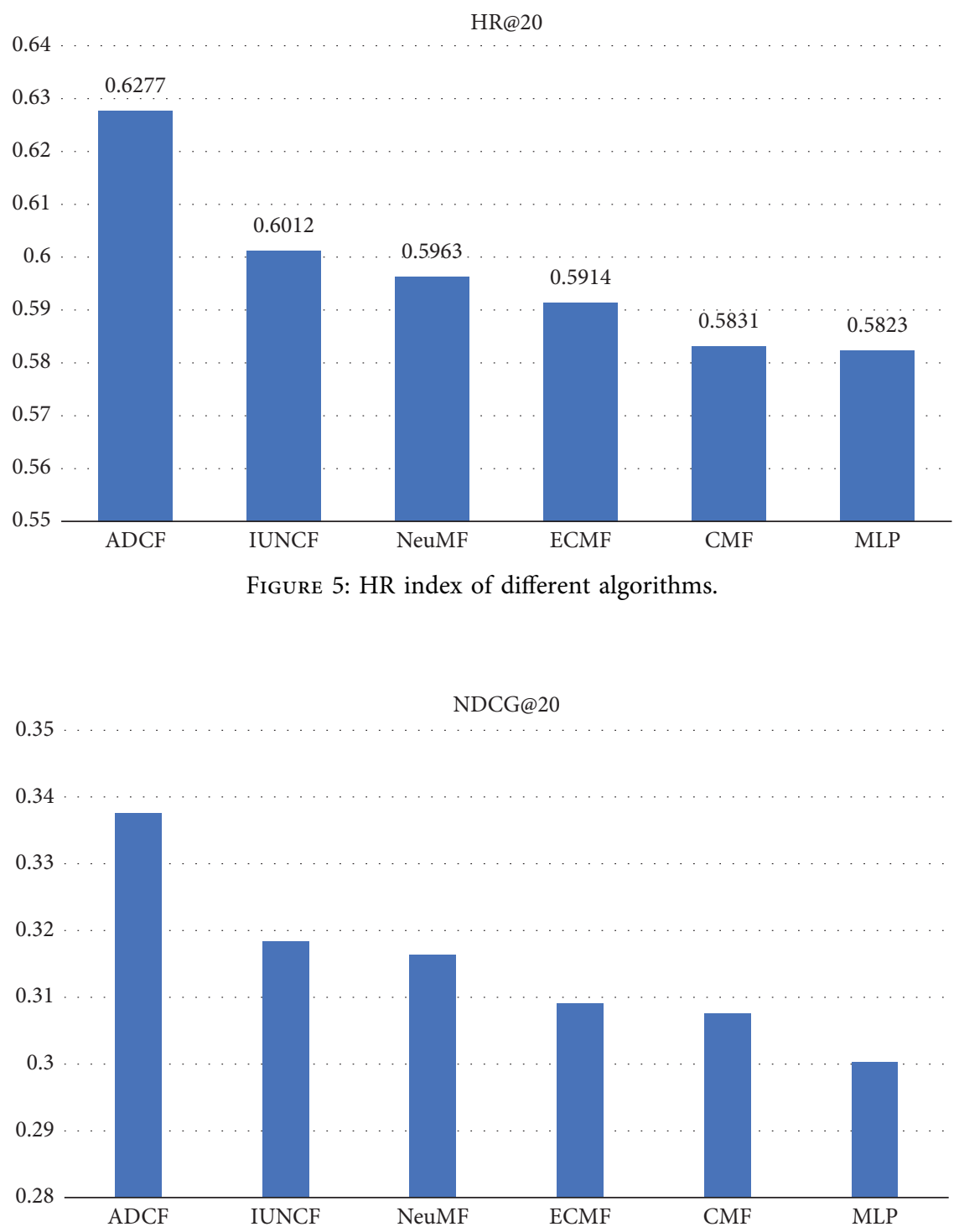

Figure 6: NDCC index of different algorithms.

to 20. In order to reduce the time complexity of model evaluation, $K$ in HR@K and NDCG@K is uniformly set to 20. The experimental results are averaged by multiple rounds.

The experimental results are shown in Figures 5 and 6 and Table 1. As can be seen from Figures 5 and 6, EGMF has improved in NDCG@20 and HR@20 indexes compared with GMF.

4.3. Comparative Experimental Selection. In order to verify the effect of the learner model (Model 1) which integrates the learner's aspect emotion and is applied to online course recommendation, this paper applies the learner model (Model 2) which does not integrate the emotion factor and the learner model which integrates the learner's emotion (Model 3) to course recommendation at the same time. To compare the advantages and
TABLE 1: Results of different algorithms in data sets.

\begin{tabular}{lccc}
\hline Different algorithms & HR@20 & NDCG@20 & t/s \\
\hline ADCF & 0.6277 & 0.3375 & $11.8+1.8$ \\
IUNCF & 0.6012 & 0.3183 & 16 \\
NeuMF & 0.5963 & 0.3163 & $12.4+0.5$ \\
ECMF & 0.5914 & 0.3091 & 8.6 \\
CMF & 0.5831 & 0.3075 & 7.8 \\
MLP & 0.5823 & 0.3003 & 10 \\
\hline
\end{tabular}

disadvantages of the three methods, the comparative experiments used in this paper are as follows:

(1) The DeepFM is used to recommend courses based on Model 2: without considering learners' emotion, the recommendation model, which is used to judge the integration of learners' emotion, can reflect learners' learning preference more accurately. 
0.95
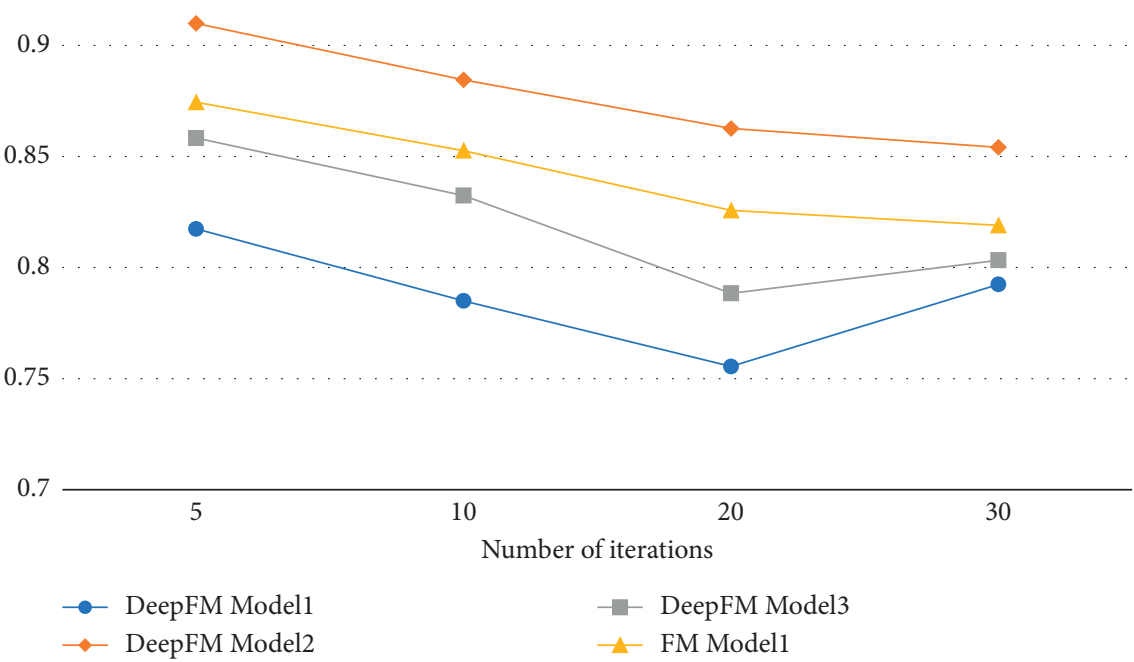

Figure 7: RMSE value.

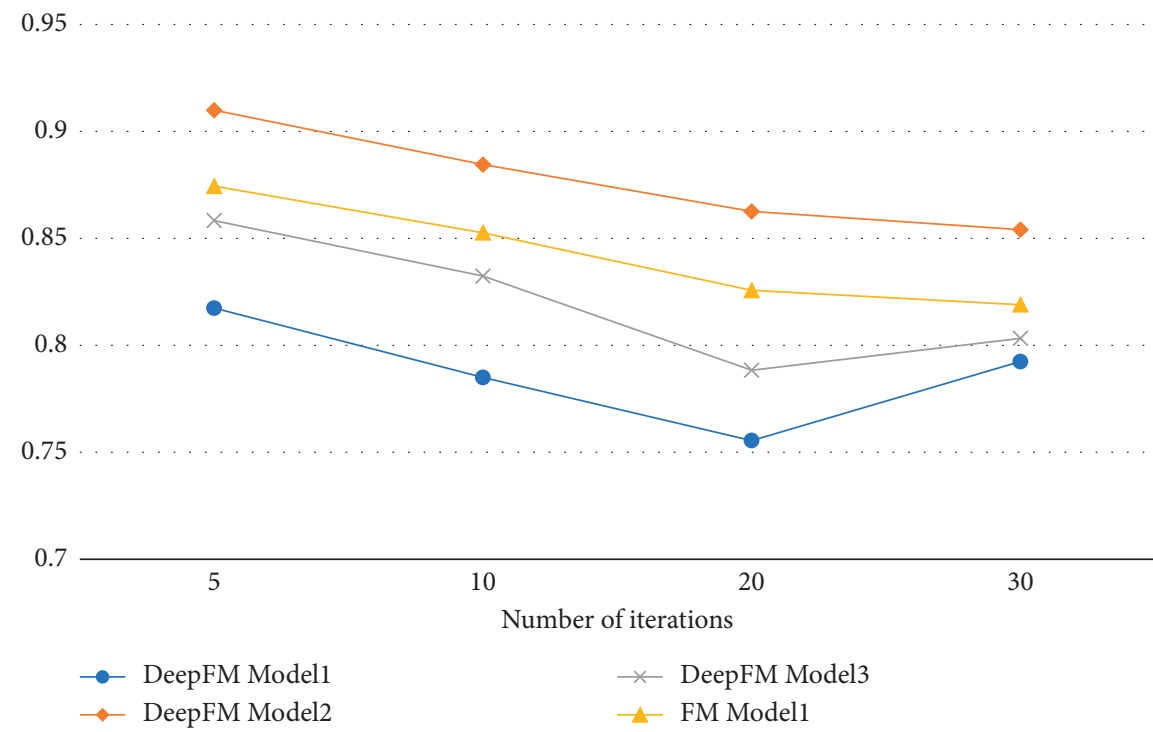

Figure 8: MAE values.

(2) Use DeepFM to recommend courses based on Model 3 : it is used to judge that the aspect emotion of integrating learners can reflect learners' preferences more carefully than the whole emotion of integrating learners only.

(3) Use FM to recommend courses based on Model 1: only using classic FM to recommend courses is used to judge the learner model constructed in this paper. Traditional recommendation technology can also achieve good results and judge that deep learning technology has higher recommendation accuracy than general FM.
4.4. Experimental Results and Analysis. Figure 7 shows the RMSE values recommended based on Model 1, Model 2, and Model 3 using a deep neural factorization machine and the RMSE values recommended based on Model 1 using a conventional FM. The experimental results show that the RMSE value changes with the increase in the number of iterations. When the number of iterations is 30 , the RMSE value recommended by both DeepFM and FM is at the minimum, and the accuracy is the highest, reaching the best state. Looking at the whole picture, DeepFM based on Model 1 recommends the smallest RMSE value, the second is the DeepFM recommendation based on Model 3, the third is the 
FM recommendation based on Model 1, and the last is the DeepFM recommendation based on Model 2, which has the lowest recommendation accuracy. So RMSE value of course recommendation based on Model 1 is smaller than that based on Model 2, while the accuracy of course recommendation based on the same learner model using DeepFM is higher than that using FM.

Figure 8 shows the RMSE values recommended based on Model 1, Model 2, and Model 3 using a deep neural factorization machine and the MAE values recommended based on Model 1 using a conventional FM. It can be seen from the table that when the number of iterations is 20 , no matter which model is based on which technology is used, the recommended MAE value based on Model 1 is generally smaller than that of Model 2, and the performance of DeepFM is generally better than that of FM based on the same learner model.

To sum up, we can draw the following conclusions:

(1) Whether DeepFM or FM is used for course recommendation, the accuracy of Model 1 in the field of course recommendation is higher than that of Model 2. The accuracy of the DeepFM course recommendation based on Model 3 is higher than that based on Model 2. Therefore, it can be concluded that learners' emotional characteristics play an extremely important role in improving the accuracy of curriculum recommendation. The learner model with emotional factors constructed in this paper perfects and makes up for the lack of emotional factors in general learner models and further perfects the learner model.

(2) Based on the same learner model, the recommendation accuracy of DeepFM is higher than that of FM. Therefore, using deep learning technology to recommend courses is helpful to improve recommendation accuracy.

(3) The recommendation accuracy of DeepFM based on Model 1 is higher than that based on Model 3. Therefore, extracting learners' aspect emotion can describe learners' preferences more carefully than extracting the whole emotion of comments, which can effectively improve the recommendation accuracy. Deep learning technology can effectively do this. Therefore, under the premise of the same learner model, DeepFM has a higher recommendation accuracy rate. However, the fine-grained analysis of emotion in this paper greatly improves the accuracy of describing learners' preferences, so the recommendation accuracy of DeepFM and FM based on Model 1 is higher than that based on Model 2 , and the recommendation accuracy of DeepFM based on Model 2 is less than that of FM based on Model 1.

\section{Conclusion}

In this paper, the learner model integrating emotional factors is applied to the field of online course recommendation, and the deep neural factorization machine is used to realize accurate and personalized course recommendation for learners. At the same time, this paper sets up a control experimental group for comparison. The experimental results are as follows. The root mean square error and average absolute error of course recommendation based on learner model with emotional factors are lower than those based on learner model without emotional factors. The learner model integrating aspect emotion has a higher recommendation accuracy rate than that integrating overall emotion of learners' comments, and the accuracy of DeepFM is higher than that of FM under the same model. This proves the effectiveness of the learner model constructed in this paper and proves the importance of learners' emotion for accurately depicting learning preferences and the advantages of deep learning technology applied to curriculum recommendation.

\section{Data Availability}

The experimental data used to support the findings of this study are available from the corresponding author upon request.

\section{Conflicts of Interest}

The author declares that there are no conflicts of interest regarding this work.

\section{Acknowledgments}

This work was supported by the Research Project of Teaching Reform in Universities of Hunan Province of China (HNJG-2020-1188) and the Natural Science Foundation of Hunan Province of China (16C0114).

\section{References}

[1] G. B. Chen, L. Wang, M. Alam, and M. Elhoseny, "Intelligent group prediction algorithm of GPS trajectory based on vehicle communication," IEEE Transactions on Intelligent Transportation Systems, vol. 22, no. 7, pp. 3987-3996, 2020.

[2] W. Qin, Y. Hu, J. Lei, and Y. Wang, "Comfort design and optimization of direct expansion multi-connected radiant air conditioning based on 3D flow field simulation," Displays, vol. 69, Article ID 102054, 2021.

[3] G. Sun, C.-C. Chen, and S. Bin, "Study of cascading failure in multisubnet composite complex networks," Symmetry, vol. 13, no. 3, p. 523, 2021.

[4] X. Ning, K. Gong, W. Li, L. Zhang, X. Bai, and S. Tian, "Feature refinement and filter network for person re-identification," IEEE Transactions on Circuits and Systems for Video Technology, vol. 31, no. 9, pp. 3391-3402, 2021.

[5] S. Mohan Krishna and T. Perumal, "Making buildings smarter and energy-efficient-using the Internet of Things platform," IEEE Consumer Electronics Magazine, vol. 10, no. 3, pp. 34-41, 2021.

[6] H. Fang, D. Zhang, Y. Shu, and G. Guo, “Deep learning for sequential recommendation: algorithms, influential factors, and evaluations," ACM Transactions on Information Systems, vol. 39, no. 1, pp. 1-42, 2020.

[7] L. P. J. Rani, D. C. J. W. Wise, K. Ajayram, T. Gokul, and B. Kirubakaran, "Course recommendation for students using 
machine learning," in Proceedings of the 2020 International Conference on Electronics and Sustainable Communication Systems (ICESC), pp. 381-384, Coimbatore, India, July 2020.

[8] M. F. Aljunid and M. D. Huchaiah, "Multi-model deep learning approach for collaborative filtering recommendation system," Journal of Intelligent Technology, vol. 5, no. 4, p. 8, 2020.

[9] A. Karatzoglou and B. Hidasi, "Deep learning for recommender systems," in Proceedings of the The Eleventh ACM Conference, pp. 815-824, ACM, Como, Italy, August 2017.

[10] M. Nakayama, K. Mutsuura, and H. Yamamoto, "Impact of learner's characters and learning behavior on learning performance during a fully online course," Electronic Journal of E-Learning, vol. 12, no. 4, pp. 394-408, 2014.

[11] D. Kim, E. Jung, M. Yoon et al., "Exploring the structural relationships between course design factors, learner committee, self-directed learning, and intentions for further learning in a self-paced MOOC," Computers \& Education, vol. 166, no. 6, pp. 104-171, 2021.

[12] X. Wang, Y. Zhang, S. Yu, X. Liu, Y. Yuan, and F.-Y. Wang, "E-learning recommendation framework based on deep learning," in Proceedings of the IEEE International Conference on Systems, pp. 455-460, IEEE, Banff, Canada, October 2017.

[13] Q. V. Dang and C. Ignat, "DTrust: a simple deep learning approach for social recommendation," in Proceedings of the 2017 IEEE Third International Conference on Collaboration and Internet Computing (CIC), pp. 209-218, IEEE, San Jose, CA, USA, October 2017.

[14] K. Cheng, X. Guo, X. Cui, and F. Shan, "Dynamical modeling, analysis, and control of information diffusion over social networks: a deep learning-based recommendation algorithm in social network," Discrete Dynamics in Nature and Society, vol. 2020, no. 4, 8 pages, Article ID 3273451, 2020.

[15] L. W. Huang, B. T. Jiang, and L. V. Shou-Ye, "Survey on deep learning based recommender systems," Chinese Journal of Computers, vol. 41, no. 7, pp. 192-219, 2018.

[16] R. Mu, "A survey of recommender systems based on deep learning," IEEE Access, vol. 6, Article ID 69009, 2019.

[17] B. Tilahun, C. Awono, and B. Batchakui, "A survey of state-ofthe-art: deep learning methods on recommender system," International Journal of Computers and Applications, vol. 162, no. 10, pp. 17-22, 2017.

[18] S. Muruganandam and N. Srininvasan, "Personalised e-learning system using learner profile ontology and sequential pattern mining-based recommendation," International Journal of Business Intelligence and Data Mining, vol. 12, no. 1, p. 78, 2017.

[19] S. R. Chavare, C. J. Awati, and S. K. Shirgave, "Smart recommender system using deep learning," in Proceedings of the Sixth International Conference on Inactive Computation Technologies [ICICT 2021], pp. 258-264, Coimbatore, India, January 2021.

[20] X. Zhang, X. Liang, A. Zhiyuli, S. Zhang, R. Xu, and B. Wu, "An attention-based LSTM model for financial time series prediction," IOP Conference Series: Materials Science and Engineering, vol. 569, no. 5, pp. 1-7, 2019.

[21] Y. Xu, H. Fang, J. Luo, J. He, T. Li, and S. Lin, "Risk evaluation method of import and export goods based on fuzzy reasoning and DeepFM," Mathematical Problems in Engineering, vol. 2021, no. 1, 8 pages, Article ID 2390958, 2021.

[22] D. Yao, S. Li, A. Li, and Y. Chen, "A recommendation method for highly sparse dataset based on teaching recommendation factorization machines," Computers, Materials \& Continua, vol. 64, no. 3, pp. 1959-1975, 2020.
[23] G. Li, J. Yuchi, H. Yang, and K. Li, "A network delay factor model based on the hidden Markov model and latent dirichlet allocation," IEEE Access, vol. 7, Article ID 133136, 2019.

[24] Y. Dong and H. Wang, "Robust output feedback stabilization for uncertain discrete-time stochastic neural networks with time-varying delay," Neural Processing Letters, vol. 51, no. 1, pp. 83-103, 2020.

[25] Y. Liu, X. Wang, X. Zhu, and Y. Zhai, "Thermal error prediction of motorized spindle for five-axis machining center based on analytical modeling and BP neural network," Journal of Mechanical Science and Technology, vol. 35, no. 1, pp. 281-292, 2021.

[26] A. Ngoc-Lan Huynh, R. C. Deo, M. Ali, S. Abdulla, and N. Raj, "Novel short-term solar radiation hybrid model: long shortterm memory network integrated with robust local mean decomposition," Applied Energy, vol. 298, no. 10, Article ID 117193, 2021.

[27] Y. Park, J. Lee, H. Moon, Y. S. Choi, and M. Rho, "Discovering microbe-disease associations from the literature using a hierarchical long short-term memory network and an ensemble parser model," Scientific Reports, vol. 11, no. 1, p. 4490, 2021.

[28] S. Surendhar and R. Vasuki, "Breast cancer detection using deep belief network by applying feature extraction on various classifiers," Turkish Journal of Computer and Mathematics Education (TURCOMAT), vol. 12, no. 1S, pp. 471-487, 2021.

[29] T. Okudono, M. Waga, and T. Sekiyama, "Weighted automata extraction from recurrent neural networks via regression on state spaces," Proceedings of the AAAI Conference on Artificial Intelligence, vol. 34, no. 4, pp. 5306-5314, 2020. 